\title{
The Water Neutron Detector
}

\author{
Alexandra (Sasha) Asghari
}

\begin{abstract}
Information gathering and dissemination is a crucial aspect of a resilient society during and after a major disruptive event. Neutron detection is particularly important when spontaneously fissioning isotopes are present, such as following a severe nuclear accident. Historically, most neutron detectors have been based on helium-3. Yet because the supply of helium-3 has greatly diminished in the past decade, it is of international interest to develop non-helium-3 based neutron detectors. The Water Neutron Detector (WaND) provides an efficient, non-toxic, and non-flammable alternative detector method. The WaND system is currently under investigation for the nondestructive assay of spent nuclear fuel to quantify plutonium content.
\end{abstract}

Keywords Nondestructive assay - Neutron detection • Water Cherenkov • IAEA • Spent fuel monitoring

\section{Introduction}

\subsection{Background}

Without an adequate, assured supply of helium-3 (or an effective replacement), IAEA safeguards in particular (and worldwide safeguards in general) will be significantly impaired. The IAEA Workshop on Requirements and Potential Technologies for Replacement of helium-3 Detectors in IAEA Safeguards Applications.

The IAEA has been involved in Japan's efforts to plan and implement decommissioning of the Fukushima Daiichi Nuclear Power Station. For example, at the Government of Japan's request, the IAEA assessed the "Mid-and-Long-Term Roadmap towards the Decommissioning of TEPCO's Fukushima Daiichi Nuclear

\footnotetext{
A. (Sasha) Asghari ( $\square)$

University of California, Berkeley, USA

e-mail: asghari@berkeley.edu 
Power Stations Units 1-4" in an effort to enhance international cooperation and sharing of information and knowledge concerning the accident and the future decommissioning process. The review focused on the safety and technological aspects of decommissioning, radioactive waste management, control of underground water, and planning of the implementation of pre-decommissioning and decommissioning activities. A major aspect of decommissioning is removal of the spent fuel $[1,2]$.

Neutron detectors are an effective technology to search for signatures of fission because natural sources of neutrons are relatively rare compared to sources of other types of radiation, such as gamma. Neutron multiplicity counters can exploit the burst-like temporal signature of fission events to reveal information about the fissioning sample. For example, the multiplication, $(\alpha, n)$ rate, and effective plutonium mass may be determined. Helium-3-based neutron detectors comprise essentially all neutron detectors currently used by the IAEA, and the US has historically been the primary supplier [3]. The U.S. stockpile of helium-3 plummeted from $\sim 230,000 \mathrm{~L}$ in 2001 to $\sim 50,000 \mathrm{~L}$ in 2010 due primarily to an increase in radiation portal monitors to combat nuclear smuggling after 9/11 [4]. Because of the connection between the $U$. S. stockpile of helium-3 and the effectiveness of IAEA inspections, it is of national and international interest to develop non-helium-3 based neutron detectors.

Furthermore, the U.S. National Nuclear Security Administration, via the Next Generation Safeguards Initiative, has identified neutron multiplicity as a priority for nondestructive assay (NDA) of spent nuclear fuel. Methods for direct and accurate measurement of plutonium content in spent fuel requiring fewer unverified a priori assumptions about the fuel matrix are needed. Plutonium measurement in spent fuel using multiplicity counting is a technically challenging problem because the gamma flux from spent fuel quickly overwhelms most neutron detectors. Such measurements would aid in quantifying shipper/receiver differences, determining the input accountability value at reprocessing facilities, and provide quantitative input to burnup credit determination for repositories [5]. Knowledge of spent fuel plutonium content in the case of a nuclear accident would aid in better decision making based on scientific data for a more resilient society.

\subsection{Novel Neutron Detector}

The WaND (Water Neutron Detector) [6] is a non-helium-3 based neutron multiplicity counter under development at Lawrence Livermore National Laboratory. It is an efficient, stable, non-toxic, and non-flammable solution to some neutron multiplicity counting applications. Neutron multiplicity refers to the number of neutrons emitted per fission event and may be used to determine the effective plutonium mass in a plutonium-bearing sample or fingerprint special nuclear material, such as plutonium or uranium. The advantage of using a neutron multiplicity counter is that the analysis is non-destructive, has the possibility of being done on-site, and is relatively fast. The WaND system is composed of $1 \mathrm{~m}^{3}$ of pure $18 \mathrm{M} \Omega$ deionized water doped 


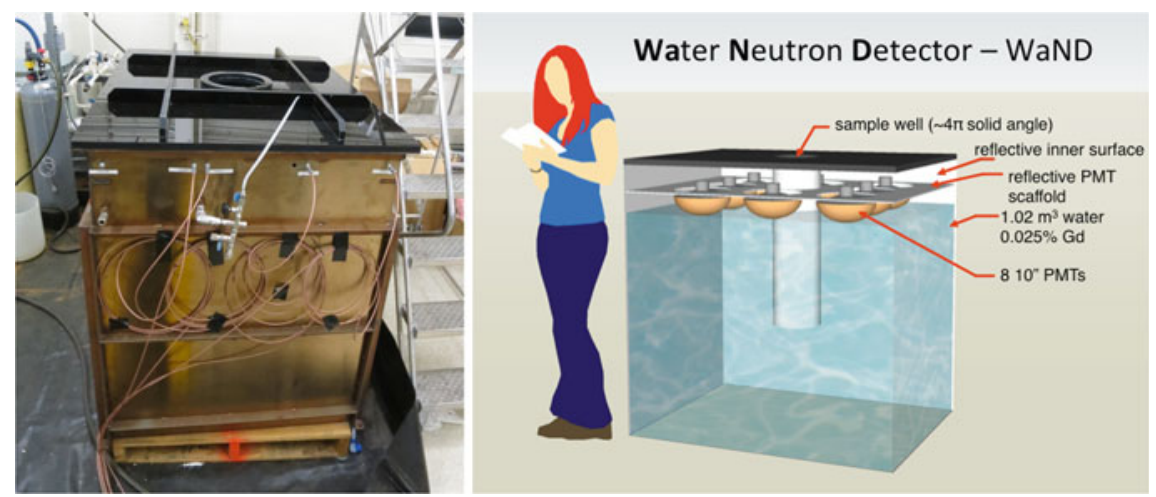

Fig. 1 A photograph (left) and 3D model created using Sketchup (right) of the WaND system. Note the person for scale

with $0.5 \%$ gadolinium-chloride $\left(\mathrm{GdCl}_{3}\right)$, contained within a stainless steel tank $(121.9 \mathrm{~cm} \times 91.4 \mathrm{~cm} \times 119.4 \mathrm{~cm})$. To protect the stainless steel tank from the corrosive water (due to the chloride content), the tank is coated with a baked-on layer of Teflon. Eight 10-in. diameter photomultiplier tubes are mounted on the top of the detector, looking into the detector volume. The inside of the tank is also lined with a $1.0 \mathrm{~mm}$ highly reflective ( $>99 \%$ in blue near UV) layer of $\mathrm{GORE}^{\circledR} \mathrm{DRP}^{\circledR}$ material. Figure 1 shows a 3D model and a photograph of the detector.

The detection mechanism is multi-stepped. A neutron born from a fission event in the sample well must enter the water volume and thermalize. The thermalization distance is $\sim 35 \mathrm{~mm}$ for $1 \mathrm{meV}$ neutrons. Once the neutron has thermalized, it will capture on a gadolinium nucleus. Gadolinium-157 (15\% natural abundance) has the highest thermal neutron absorption cross-section of any stable isotope $(259,000$ barns), and natural gadolinium's thermal neutron absorption cross section is 50,000 barns. Upon capture, the target nucleus enters an $8 \mathrm{meV}$ excited state, then de-excites by emitting a gamma cascade with a total energy of $8 \mathrm{meV}$. The gammas then Compton scatter electrons in the water, ejecting some at high energies. Electrons that are scattered with a kinetic energy above the Cherenkov threshold (250 keV [7]) will produce a ring of Cherenkov light, which is then detected by the photomultiplier tubes.

\section{Results}

Both gammas and neutrons produce a detector response in the WaND system. Figure 2 shows the spectral response of a 5.9 microCi cobalt- 60 and a 0.82 microCi californium-252 source. The background spectrum is measured by performing a data run without sources. Then the background is statistically subtracted from a source run, leaving only the source contribution. 


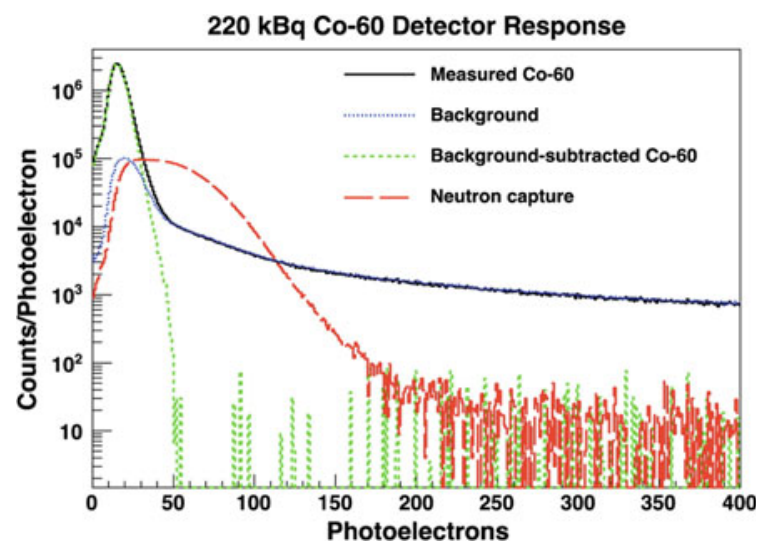

Fig. 2 The detector response spectrum from a $1 \mathrm{~h}$ run with a 5.9 microCurie cobalt-60 gamma and a 0.82 microCurie californium-252 neutron source [6]. The solid black line indicates the cobalt-60 spectrum prior to background subtraction. The dotted blue line is a no-source background spectrum. The dashed green line shows the statistical subtraction of the cobalt-60 source and background, which leaves the pure cobalt-60 detector response spectrum. The dashed red line shows a pure neutron californium-252 spectrum, also background subtracted

Cobalt-60 is used by national and international organizations as a standard candle for gamma rejection. In our detector, cobalt-60 is a proxy for unwanted low energy background gamma rays that may be associated with a source. Figure 2 shows that it is possible to remove nearly all of these gamma rays by applying a 50 photoelectron energy cut. The absolute neutron detection efficiency with this criteria is $28 \%$, while only 1 part in $10^{8}$ of the background remains. This translates to a sensitivity to $\sim 20$ to $30 \mathrm{mg}$ of plutonium-240 by measuring the multiplicity distribution [8].

\section{Conclusions and Future Work}

The WaND system is currently under investigation for possible application to spent fuel monitoring. Spent fuel poses an especially difficult problem for neutron detection because the high intensity gamma field renders most neutron detectors useless. For example, scintillator-based neutron detector systems rely on pulse shape discrimination, placing severe limits on gamma background and neutron signal rate. Germanium or silicon-based detectors are small and susceptible to neutron damage. Boron-based systems such as $\mathrm{BF}_{3}$ and ${ }^{10} \mathrm{~B}$ tubes and planes present toxicity concerns and can be relatively inefficient. The WaND system is efficient, stable, non-toxic, and non-flammable. The effects of high gamma fields and how to mitigate them are currently under investigation. Future work includes experimentally measuring the multiplicity distribution of a plutonium source. The 
combination of plutonium sensitivity and gamma insensitivity creates a potential to directly measure the plutonium content in spent fuel. In the case of a severe nuclear accident, knowing the content nuclear contamination will aid decision makers in making science-driven decisions on how to better recover.

Acknowledgements The author would like to thank Karl van Bibber, Adam Bernstein, and Steven Dazeley for their mentorship and infinite patience. This material is based upon work supported by the National Science Foundation Graduate Research Fellowship under Grant No. DE-NA0000979.

\section{References}

1. International Atomic Energy Agency, Preliminary Summary Report: The IAEA International Peer Review Mission on Mid-and-Long-Term Roadmap Towards the Decommissioning of TEPCO's Fukushima Daiichi Nuclear Power Stations Units 1-4, Tokyo and Fukushima Prefecture, Japan (2015)

2. IAEA Team Completed Third Review of Japan's Plans to Decommission Fukushima Daiichi. International Atomic Energy Agency, 17 Feb 2015, Web

3. M.M. Pickrell, The IAEA workshop on requirements and potential technologies for replacement of helium-3 detectors in IAEA safeguards applications. J. Nucl. Mater. Manag. 41(2), 14-29 (2013)

4. D.A. Shea, D. Morgan, The helium-3 shortage: supply, demand, and options for congress, Congressional Research Service (2010). www.crs.gov

5. S.J. Tobin, et al., Next generation safegaurds initiative research to determine the Pu mass in spent fuel assemblies: purpose, approach, constraints, implementation, and calibration. NIMA 652, 73-75 (2011)

6. S. Dazeley, A. Asghari, A. Bernstein, N. Bowden, V. Mozin, A water-based neutron detector as a well multiplicity counter. NIMA 771(0), 32-38 (2015). doi:http://dx.doi.org/ 10.1016/j. nima.2014.10.028

7. G.S. Mitchell, R.K. Gill, D.L. Boucher, C. Li, S.R. Cherry. doi:10.1098/rsta.2011.0271 (Published 28 November 2011)

8. S. Dazeley, et al., Performance characterization of a water-based multiplicity counter, in 55th INMM Annual Meeting in Atlanta, GA. 2014 (for a Journal or Transactions Summary. Trans. Am. Nucl. Soc. 98, 1200 (2008))

Open Access This chapter is licensed under the terms of the Creative Commons Attribution 4.0 International License (http://creativecommons.org/licenses/by/4.0/), which permits use, sharing, adaptation, distribution and reproduction in any medium or format, as long as you give appropriate credit to the original author(s) and the source, provide a link to the Creative Commons license and indicate if changes were made.

The images or other third party material in this chapter are included in the chapter's Creative Commons license, unless indicated otherwise in a credit line to the material. If material is not included in the chapter's Creative Commons license and your intended use is not permitted by statutory regulation or exceeds the permitted use, you will need to obtain permission directly from the copyright holder.

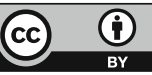

